\title{
The Prevalence of Entamoeba Histolytica and Giardia Lamblia in Nasiryah city: A household survey
}

\author{
Dr. Abdulreda Abed Hatam, M.B.Ch.B. D.C.M. Almosawi Pediatric Hospital
}

\section{$\underline{\text { Abstract }}$}

\section{Background}

Despite the prevailing impression among many professional that parasitic infection is very common in our population, and despite the usual blame we put on parasitic infections as a cause of ill-health, yet, scientific studies to document such a problem are scanty. In Thi-Gar governorate, the situation is not exceptional. This study is an attempt to quantify the prevalence of two important parasitic infections : namely Entamoeba histolytica and Giardia lamblia in Nasiryiah city, and to describe the relationship of prevalence of these infections to selected socioeconomic variables.

\section{Methods}

The study is cross-sectional household survey, it was conducted during April and May of 2019.Two districts (Aledaralmahalia and Alsader town) were selected in Nasiryah city. The selection depend upon the difference in socioeconomic status, assuming that they represent the wide spectrum of socioeconomic variation in the city population, but it must be admitted that this only assumption and no more.

\section{Results}

There are generally many difference between the two districts.Aledaralmahalia has relatively better sanitation, people live in better housing conditions, they are moderately educated and $16 \%$ of houses are rented. Alsader town has poorer sanitation, over-crowded housing conditions and $28 \%$ of houses are rented. The prevalence rates are higher in Alsader town(E.H.15.09\%,G.L.11.09\%), than those in Aledaralmahalia (E.H.11.18\%,G.L.10.4\%).

\section{Conclusion}

The result of study revealed that the prevalence rates of Entamoeba histolytica and Giardia lamblia were higher in Alsader town than in Aledaralmahalia.

Keywords: prevalence, Entamoeba, Giardia . 
Web Site: https://jmed.utq.edu.iq

ISSN (Print):1992-92 18, ISSN (Online):1992-92 18

DOI: https://doi.org/10.32792/utq/utjmed/19/1/5
Email:utjmed@utq.edu.iq

\section{Introduction}

\section{Amoebiasis}

Is caused by Entamoeba histolytica, which is spread between humans by its cysts. It is one of the leading parasitic causes of morbidity and mortality in the topics and is occasionally acquired in non tropical countries(1). Clinical syndromes associated with Entamoeba histolytica infection include noninvasive intestinal infection, intestinal amoebiasis, ameboma, and liver abscess. Disease is more severe in the very young, the elderly, and pregnant women(2).

\section{Giardiasis}

Giardia occur worldwide and it is the most common intestinal parasite identified in the puplic health laboratories. The age specific prevalence of of Giardiasis is high during the childhood and begin to decline after adolescence. Asymptomatic carriage may persist for several months(3). Giardia parasite transmission occurs through ingestion of faecally contaminated food or water,or through person to person contact. Symtoms Include prolonged diarrhea, abdominal pain, malabsorption, bloating, dehydration and weight loss(4).

\section{Background}

Amoebiasis is thought to occur in travellers, migrants from endemic areas,and among men who have sex with men. Amoebiasis caused by Entamoeba histolytica, has worldwide distribution, with an estimated 30 million people being infected(5).

Giardiasis has global distribution and it is a common cause of diarrhea in both children and adults and is transmitted via the faecal-oral route through direct or indirect ingestion of cysts. The prevalence of Giardia infection is higher in developing countries(6).

Girdia lamblia and Entamoeba histolytica are important pathogenic intestinal parasites and among the leading causes worldwide of diarrheal illness in humans. Diseases caused by these organism, are characterized by self-limited diarrhea, but can evolve to longterm complication(7).

Diagnosis of Amoebiasis present difficulties, particularly in epidemiological surveys, because the microscopical techniques used require highly skilled personal seldom available where infection is most prevalent.Giardiasis frequently coexist with Amoebiasis and is transmitted by the same way. Diagnosis of Giardiasis requires skilled microscopy, and false negative tests are common because cysts are excreted in the stool irregularly(8).

\section{Methods}

Epidemiological, socioeconomic, and demographic variables were obtained by using special questionnaire. The work was done by the investigator and three assistants. Ten households were visited daily. The information was registered, and fresh stool specimens were collected on the next morning from those households. 100 households were selected from each district. All specimens were examined by one laboratory assistant, and $10 \%$ of these specimens were reexamined by the investigator, as a measure of cross checking.

The population in the two districts had different level of income,education, occupation, and value of dwelling places. All ages and both sexes were examined. The main items in the questionnaire were serial number 
Thi-Qar Medical Journal (TQMJ):Vol.(19), No.(1), 2020

Web Site: https://jmed.utq.edu.iq

ISSN (Print):1992-92 18, ISSN (Online):1992-92 18

DOI: https://doi.org/10.32792/utq/utjmed/19/1/5

of the household, name of the district, name of the head of the household, type of the house, source of water supply, presence or absence of latrines, presence or absence of animals, presence of household gadgets, house ownership, and the family size.

\section{Results}

There were generally many differences between the two districts. Aledaralmahalia had relatively better sanitation, people lived in better housing condition, they are moderately educated, and $16 \%$ of houses were rented. Alsader town had poorer sanitation,people lived in poor and over-crowded housing condition, $28 \%$ of houses were rented, and animal were present in $2 \%$ of the households.
Email:utjmed@utq.edu.iq

The results were tabulated in six tables. In general, the prevalence rate of Entamoeba histolytica in Alsader town $(15.09 \%)$ is higher than that in Aledaralmahalia (11.13\%).The prevalence rate of Giardia lamblia in Alsader town $(11.07 \%)$ is higher than that in Aledaralmahalia (10.4\%).

\begin{tabular}{|c|c|c|c|c|c|c|c|c|c|c|c|c|c|c|c|c|c|c|}
\hline \multirow[b]{3}{*}{ Age } & \multicolumn{9}{|c|}{ Aledaralmahalia } & \multicolumn{9}{|c|}{ Alsader town } \\
\hline & \multicolumn{3}{|c|}{ Male } & \multicolumn{3}{|c|}{ Femal } & \multicolumn{3}{|c|}{ Total } & \multicolumn{3}{|c|}{ Male } & \multicolumn{3}{|c|}{ Femal } & \multicolumn{3}{|c|}{$\overline{\text { Total }}$} \\
\hline & $\begin{array}{c}\text { no.e } \\
x .\end{array}$ & $\begin{array}{l}\text { no. } \\
\text { inf. }\end{array}$ & $\%$ & \begin{tabular}{|c} 
no.e \\
$x$.
\end{tabular} & $\begin{array}{l}\text { no. } \\
\text { inf. }\end{array}$ & $\%$ & \begin{tabular}{|c} 
no.e \\
$x$.
\end{tabular} & $\begin{array}{l}\text { no. } \\
\text { inf. }\end{array}$ & $\%$ & $\begin{array}{c}\text { no.e } \\
x .\end{array}$ & $\begin{array}{l}\text { no. } \\
\text { inf. }\end{array}$ & $\%$ & $\begin{array}{c}\text { no.e } \\
x .\end{array}$ & $\begin{array}{l}\text { no. } \\
\text { inf. }\end{array}$ & $\%$ & $\begin{array}{c}\text { no.e } \\
x .\end{array}$ & $\begin{array}{l}\text { no. } \\
\text { inf. }\end{array}$ & $\%$ \\
\hline$<1 y$ & 6 & 0 & 0 & 5 & 0 & 0 & 11 & 0 & 0 & 4 & 0 & 0 & 5 & 0 & 0 & 9 & 0 & 0 \\
\hline $1-<5$ & 30 & 2 & 6.76 & 33 & 3 & 9.09 & 63 & 5 & 7.94 & 30 & 0 & 0 & 27 & 0 & 0 & 57 & 0 & 0 \\
\hline $5-<15$ & 97 & 12 & 13.37. & 74 & 4 & 5.4 & 171 & 16 & 9.36 & 76 & 8 & 10.53 & 64 & 9 & 14.06 & 140 & 17 & 12.19 \\
\hline $15-<45$ & 106 & 14 & 13.29 & 112 & 17 & 15.18 & 218 & 31 & 14.22 & 65 & 16 & 29.61 & 101 & 18 & 17.82 & 166 & 34 & 20.48 \\
\hline $45+$ & 47 & 5 & 10.63 & 38 & 4 & 10.53 & 85 & 9 & 10.52 & 22 & 5 & 22.72 & 30 & 8 & 26.67 & 52 & 13 & 25 \\
\hline total & 286 & 33 & 11.54 & 262 & 28 & 10.69 & 548 & 61 & 11.13 & 197 & 29 & 14.72 & 227 & 35 & 15.41 & 424 & 64 & 15.7 \\
\hline
\end{tabular}

Table No.1 age and sex distribution of population with respect to Entamoeba hitolytica infection 
Thi-Qar Medical Journal (TQMJ):Vol.(19), No.(1), 2020

Web Site: https://jmed.utq.edu.iq

Email:utjmed@utq.edu.iq

ISSN (Print):1992-92 18, ISSN (Online):1992-92 18

DOI: https://doi.org/10.32792/utq/utjmed/19/1/5

\begin{tabular}{|c|c|c|c|c|c|c|c|c|c|c|c|c|c|c|c|c|c|c|}
\hline \multirow[b]{3}{*}{ Age } & \multicolumn{9}{|c|}{ Aledralmahalia } & \multicolumn{9}{|c|}{ Alsader town } \\
\hline & \multicolumn{3}{|c|}{$\overline{\text { Male }}$} & \multicolumn{3}{|c|}{ Femal } & \multicolumn{3}{|c|}{ Total } & \multicolumn{3}{|c|}{ Male } & \multicolumn{3}{|c|}{ Femal } & \multicolumn{3}{|c|}{ Total } \\
\hline & $\begin{array}{c}\text { no.e } \\
x\end{array}$ & $\begin{array}{l}\text { no. } \\
\text { inf. }\end{array}$ & $\%$ & $\begin{array}{c}\text { no.e } \\
x\end{array}$ & \begin{tabular}{|l|} 
no. \\
inf.
\end{tabular} & $\%$ & $\begin{array}{c}\text { no.e } \\
x\end{array}$ & $\begin{array}{l}\text { no. } \\
\text { inf. }\end{array}$ & $\%$ & \begin{tabular}{|c|} 
no.e \\
$x$ \\
\end{tabular} & $\begin{array}{l}\text { no. } \\
\text { inf. }\end{array}$ & $\%$ & \begin{tabular}{|c|} 
no.e \\
$x$
\end{tabular} & $\begin{array}{l}\text { no. } \\
\text { inf. }\end{array}$ & $\%$ & $\begin{array}{c}\text { no.e } \\
x\end{array}$ & $\begin{array}{l}\text { no. } \\
\text { inf. }\end{array}$ & $\%$ \\
\hline$<1 y$ & 6 & 1 & 16.67 & 5 & 0 & 0 & 11 & 1 & 9.09 & 4 & 0 & 0 & 5 & 0 & 0 & 9 & 0 & 0 \\
\hline $1-<5$ & 30 & 3 & 10 & 33 & 3 & 9.09 & 63 & 6 & 9.52 & 30 & 1 & 3.33 & 27 & 3 & 11.11 & 57 & 4 & 7.01 \\
\hline $5-<15$ & 97 & 9 & 9.28 & 74 & 10 & 13.5 & 171 & 19 & 11.11 & 76 & 6 & 7.89 & 64 & 12 & 18.75 & 140 & 18 & 12.86 \\
\hline $15-<45$ & 106 & 9 & 8.49 & 112 & 17 & 15.18 & 218 & 26 & 11.92 & 65 & 5 & 7.69 & 101 & 13 & 12.87 & 166 & 18 & 10.84 \\
\hline $45+$ & 47 & 0 & 0 & 38 & 5 & 13.16 & 85 & 5 & 5.88 & 22 & 3 & 13.63 & 30 & 4 & 13.33 & 52 & 7 & 13.46 \\
\hline total & 268 & 22 & 7.69 & 226 & 33 & 13.36 & 548 & 57 & 10.4 & 197 & 15 & 7.6 & 227 & 32 & 14.09 & 424 & 47 & 11.09 \\
\hline
\end{tabular}

Table no.2 age and sex distribution of population with respect to Giardia lamblia infection

\begin{tabular}{|c|c|c|c|c|c|c|c|c|c|c|}
\hline \multirow[b]{3}{*}{ Education } & \multicolumn{5}{|c|}{ Aledralmahalia } & \multicolumn{5}{|c|}{ Alsader town } \\
\hline & \multirow[b]{2}{*}{ No. ex. } & \multicolumn{2}{|c|}{ E. hist } & \multicolumn{2}{|c|}{ G. Lamb. } & \multirow[b]{2}{*}{ No. ex. } & \multicolumn{2}{|c|}{ E. hist } & \multicolumn{2}{|c|}{ G. Lamb. } \\
\hline & & $\begin{array}{c}\text { No. } \\
\text { infect. }\end{array}$ & $(\%)$ & $\begin{array}{c}\text { No. } \\
\text { infect. }\end{array}$ & $(\%)$ & & $\begin{array}{c}\text { No. } \\
\text { infect. }\end{array}$ & $(\%)$ & $\begin{array}{c}\text { No. } \\
\text { infect. }\end{array}$ & $(\%)$ \\
\hline \begin{tabular}{|c|} 
children \\
$<6 y$
\end{tabular} & 98 & 7 & 7.14 & 13 & 13.26 & 84 & 2 & 2.38 & 4 & 4.76 \\
\hline illitrate & 90 & 12 & 13.33 & 5 & 5.56 & 109 & 24 & 22 & 14 & 18.84 \\
\hline $\begin{array}{c}\text { read and } \\
\text { write }\end{array}$ & 81 & 13 & 16.05 & 9 & 11.11 & 57 & 13 & 22.8 & 9 & 15.79 \\
\hline $\begin{array}{r}\text { primary } \\
\text { school } \\
\end{array}$ & 150 & 14 & 9.33 & 15 & 0 & 98 & 15 & 15.3 & 14 & 14.29 \\
\hline $\begin{array}{c}\text { intermedia } \\
\text { te scool } \\
\end{array}$ & 79 & 10 & 12.66 & 8 & 10.13 & 59 & 9 & 15.25 & 4 & 6.78 \\
\hline $\begin{array}{c}\text { preparatar } \\
\text { y school }\end{array}$ & 33 & 3 & 9.09 & 5 & 15.15 & 14 & 1 & 7.14 & 1 & 7.14 \\
\hline \begin{tabular}{|c|} 
college\& \\
institute
\end{tabular} & 17 & 2 & 11.76 & 2 & 11.76 & 3 & 0 & 0 & 1 & 33.33 \\
\hline total & 518 & 61 & 11.13 & 57 & 10.4 & 424 & 64 & 15.09 & 47 & 11.8 \\
\hline
\end{tabular}

Table no.4 Distribution of infection according to educational state of individuals 
Thi-Qar Medical Journal (TQMJ):Vol.(19), No.(1), 2020

Web Site: https://jmed.utq.edu.iq

Email:utjmed@utq.edu.iq

ISSN (Print):1992-92 18, ISSN (Online):1992-92 18

DOI: https://doi.org/10.32792/utq/utjmed/19/1/5

\begin{tabular}{|c|c|c|c|c|c|c|c|c|c|c|c|c|}
\hline \multirow[b]{3}{*}{$\begin{array}{c}\text { Occupation of } \\
\text { head of } \\
\text { household }\end{array}$} & \multicolumn{6}{|c|}{ Aledaralmahalia } & \multicolumn{6}{|c|}{ Alsader town } \\
\hline & \multirow[b]{2}{*}{$\begin{array}{c}\text { No. of } \\
\text { house-hold }\end{array}$} & \multirow[b]{2}{*}{ No. ex. } & \multicolumn{2}{|c|}{ E. hist } & \multicolumn{2}{|c|}{ G. Lamb. } & \multirow[b]{2}{*}{$\begin{array}{c}\text { No. of } \\
\text { house-hold }\end{array}$} & \multirow[b]{2}{*}{ No. ex. } & \multicolumn{2}{|c|}{ E. hist } & \multicolumn{2}{|c|}{ G. Lamb. } \\
\hline & & & $\begin{array}{l}\text { No. } \\
\text { infect. }\end{array}$ & $(\%)$ & $\begin{array}{l}\text { No. } \\
\text { infect }\end{array}$ & (\%) & & & $\begin{array}{l}\text { No. } \\
\text { infect }\end{array}$ & $(\%)$ & $\begin{array}{l}\text { No. } \\
\text { infect }\end{array}$ & $(\%)$ \\
\hline upper & 14 & 94 & 11 & 11.71 & 14 & 14.89 & 0 & 0 & 0 & 0 & 0 & 0 \\
\hline middle & 66 & 351 & 36 & 10.26 & 32 & 9.12 & 33 & 142 & 17 & 11.97 & 11 & 7.75 \\
\hline lower & 20 & 103 & 14 & 13.57 & 11 & 10.68 & 67 & 282 & 47 & 16.62 & 36 & 12.77 \\
\hline total & 100 & 548 & 61 & 11.13 & 57 & 10.4 & 100 & 424 & 46 & 15.09 & 47 & 11.8 \\
\hline
\end{tabular}

Table no.5 Distribution of infection by occupation

\begin{tabular}{|c|c|c|c|c|c|c|c|c|c|c|c|}
\hline \multirow{3}{*}{$\begin{array}{l}\text { no. of members } \\
\text { of household }\end{array}$} & \multicolumn{6}{|c|}{ Aledaralmahalia } & \multicolumn{5}{|c|}{ Alsader town } \\
\hline & \multirow{2}{*}{$\begin{array}{c}\text { no.of } \\
\text { households }\end{array}$} & \multirow{2}{*}{$\begin{array}{l}\text { No. of } \\
\text { infection }\end{array}$} & \multicolumn{2}{|c|}{ E. hist } & \multicolumn{2}{|c|}{ G. Lamb. } & \multirow{2}{*}{$\begin{array}{c}\text { No. of } \\
\text { house-hold }\end{array}$} & \multicolumn{2}{|c|}{ E. hist } & \multicolumn{2}{|c|}{ G. Lamb. } \\
\hline & & & $\begin{array}{c}\begin{array}{c}\text { No. of house- } \\
\text { hold }\end{array} \\
\end{array}$ & $(\%)$ & $\begin{array}{l}\text { No. of house- } \\
\text { hold }\end{array}$ & $(\%)$ & & $\begin{array}{l}\begin{array}{l}\text { No. of house- } \\
\text { hold }\end{array} \\
\end{array}$ & $(\%)$ & $\begin{array}{c}\begin{array}{c}\text { No. of house- } \\
\text { hold }\end{array} \\
\end{array}$ & $(\%)$ \\
\hline \multirow{3}{*}{$<5$} & \multirow{3}{*}{17} & no infect & 9 & 52.49 & 10 & 58.82 & \multirow{3}{*}{21} & 11 & 25.38 & 13 & 61.9 \\
\hline & & 1 infect & 8 & 47.05 & 7 & 41,18 & & 10 & 47.61 & 6 & 28.52 \\
\hline & & $2+$ infect & 0 & 0 & 0 & 0 & & 0 & 0 & 2 & 9.52 \\
\hline \multirow{3}{*}{$5-<10$} & \multirow{3}{*}{79} & no infect & 32 & 40.5 & 37 & 46.84 & \multirow{3}{*}{75} & 27 & 40.3 & 43 & 56.47 \\
\hline & & 1 infect & 44 & 55.7 & 39 & 49.37 & & 43 & 56.58 & 30 & 39.47 \\
\hline & & $2+$ infect & 3 & 3.8 & 3 & 3.8 & & 5 & 6.58 & 2 & 2.63 \\
\hline \multirow{3}{*}{$10_{+}$} & \multirow{3}{*}{4} & no infect & 1 & 25 & 1 & 25 & \multirow{3}{*}{4} & 3 & 75 & 1 & 25 \\
\hline & & 1 infect & 2 & 50 & 1 & 25 & & 1 & 25 & 3 & 75 \\
\hline & & $2+$ infect & 1 & 25 & 2 & 50 & & 0 & 0 & 0 & 0 \\
\hline \multirow{3}{*}{ total } & \multirow{3}{*}{100} & no infect & 42 & 42 & 48 & 48 & \multirow{3}{*}{100} & 41 & 41 & 57 & 57 \\
\hline & & 1 infect & 54 & 54 & 47 & 47 & & 54 & 54 & 39 & 39 \\
\hline & & $2+$ infect & 4 & 4 & 5 & 5 & & 5 & 5 & 4 & 4 \\
\hline
\end{tabular}

Table No.6 Clustering of infection at a household level 
Thi-Qar Medical Journal (TQMJ):Vol.(19), No.(1), 2020

Web Site: https://jmed.utq.edu.iq

ISSN (Print):1992-92 18, ISSN (Online):1992-92 18

DOI: https://doi.org/10.32792/utq/utjmed/19/1/5
Email:utjmed@utq.edu.iq

\section{Discussion}

Stool examination has its merit in its simplicity, and therefore has an important place in the diagnosis of parasitic infection, especially in areas with high prevalence rates and limited diagnostic facilities. One sample for detection of parasitic infection is not enough, especially for Giardia lamblia, because of the periodicity of fecal excretion of parasites $(9)$

The laboratory assistant examined stool specimens for ten households(about 60 samples) daily, therefore it is impossible to exclude within observer variation. The study was a cross-sectional, and was carried out within two months, ten households were interviewed daily, so it is impossible to cover all the members of households.Across-sectional study among north east Indian population showed the prevalence of Amoebiasis was 13.7\%(10).

An observational study was conducted to estimate the prevalence of Giardia infection in households of confirmed Giardia cases in nine local authorities in Lancashine between June 2014 and June 2015.Giardia was detected in $19 \%$ of households members of whom $90 \%$ were asymptomatic(11).

1520 stool samples were collected from Kadhimiyah hospital for the period between September-October 2012 and the result concentrated on the prevalence of Entamoeba histolytica(9.8\%) and Giardia lamblia(1.77\%)(12)

A study was conducted at the outpatient department of Provincial general hospital , Embu kenga. The results of the study showed that infection with Girdiasis is apparent in children aged 5 years and below(7.98\%)(13).

In a study carried in highland village in Guntemala, over $20 \%$ of the children had Giardiasis at 36 months of age(14).

Prevalence of intestinal parasites were estimated in Abha,Saudi Arabia. The prevalence of Giardiasis among te urban (3.6\%) versus the rural population (11.81\%).The prevalence of Amoebiasis among the urban was(1.7\%)(15).

\section{Conclusion}

1-The prevalence rates of Entamoeba histolytica and Giardia lamblia infections were higher in Alsader town than in Aledaralmahalia.

2-The prevalence rates of infections among females were higher than that among males, except the prevalence rate of Entamoeba histolytica in Aledaralmahalia.

3-The relation between the socioeconomic status and the infections was evident, except for educational level.

\section{Recommendations}

1-The health authorities must play an active role in prevention and control the Entamoeba histolytica and Girdia lamblia.

2-Educate the general public on personal hygiene.

3-Protect public water supply from fecal contamination and sanitary disposal of human faeces.

4-Supervision by health agencies of sanitary 
Thi-Qar Medical Journal (TQMJ):Vol.(19), No.(1), 2020

Web Site: https://jmed.utq.edu.iq

Email:utjmed@utq.edu.iq

ISSN (Print):1992-92 18, ISSN (Online):1992-92 18

DOI: https://doi.org/10.32792/utq/utjmed/19/1/5

practices of person preparing and serving food

in public eating places.

\section{References}

1-Staurt H.Ralston,Ian D,Penman,Mark W.J.Strachan,Richard P.Hobson.Davidson's principles and practice of medicine.2018,p.286.

2-Red book,American Academy of pediatric.2006,p.204.

3-Robet M.Kliegman,MD.Bonita F.Stanton,MD.Joseph W.St.Geme Ill,MD.Nino F.Shor,MD,PHD.Richard E.Behrman,MD.Nelson Textbook of pediatrics,2011,page 1181.

4-Kadyn D.Beer,Sarah A.Ccollier,Fan Du,and Julia W.Gargano.Clin.infect.Dis. 2017,May,64(9).1244-1250.

5-Ana Domazrtovska,Roganlee,Chanra Adhikari,Mathew Watts,Nicole Gilory,Damien Stark,and Shobini Sivagnonam.Tropical medicine and infectious disease.2018 Sep.3(3) 73.

6-Hossein Hooshyar,Parvin Rostamkhani,Mohsen Arbabi,and Mahdi Delavari.Gastroenterology and Hepatology from Bed to Bench.2019 winter 12(1):3-12.

7-Bruno M,Di Genova,and Renata R.Tonelli.Front.Microbiol.03 March 2016.

8-WHO Model prescribing information:Drugs used in parasitic diseases:second edition(1995,152 pages)..

9-Kamath K.P.and Mupngasu R.A.Comparative study of four methods for detection Giardia lamblia/Gastroenterology 1974:66:16-21.

10-Jvobrato Nath.Samkar Kumar Chosh,Baby Singha and Jaishree Paul.Plos Neglected Tropical Diseases.2015 Dec.9(12).

11-Atison Weldrani,Roberto Vivacos,Catherine Hartly and Knneth Lamden.BMC Infectious diseases.2017.17.468.

12-Amjed Qeys Ibrahim.The Iraqi J.Vet.Med.36(1):32-36:2012.

13-J.Thingo,O.Mucheru,F.Muite,B.Langat,P.Kamau and L.Irexi.Researh Journal of Parasitology.Volume 6(4):136-143,2011.

14-Stevens,D.P.Giardiasis host-pathogen biology.Review of infectious diseases 1982:4:859-866.

15-AL-Medani A.A.,Omer M.S..Abu-zed H.A.,and Abdulla S.A.Intestinal parasites in urban and rural communities of Abha.Annals of Saudi Medicine 1989:9:182-185. 
Web Site: https://jmed.utq.edu.iq

ISSN (Print):1992-92 18, ISSN (Online):1992-92 18

DOI: https://doi.org/10.32792/utq/utjmed/19/1/5
Email:utjmed@utq.edu.iq

\section{نسب انتشار الزرحار الامبيبي والجيارديا اللمبلية في مدينة الناصرية (دراسة عرضانية مقطعية) عبد الرضا عبد حاتم}

هذا التقرير يتضمن دراسة عرضائية مقطعية أجريت على عينة من فئن

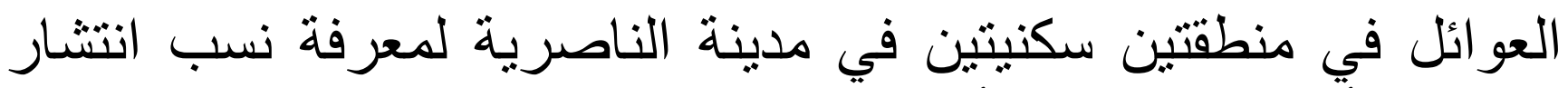

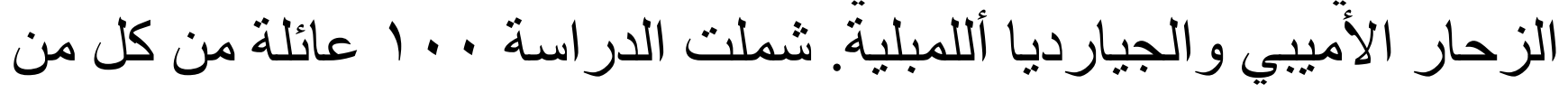

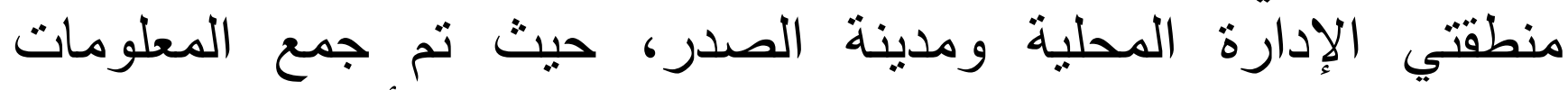

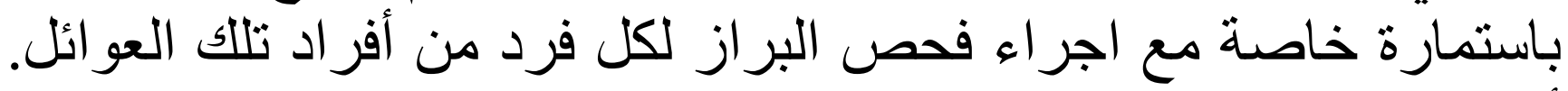

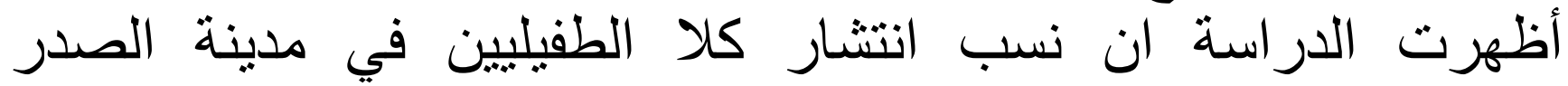

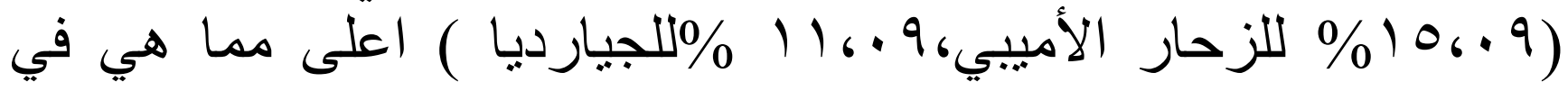

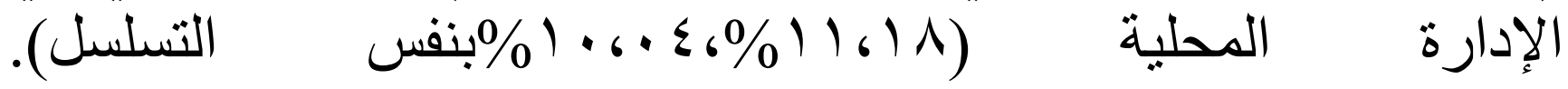

